\title{
THE MINIMAL DEGENERATION SINGULARITIES IN THE AFFINE GRASSMANNIANS
}

\author{
ANTON MALKIN, VIKTOR OSTRIK, AND MAXIM VYBORNOV
}

\begin{abstract}
The minimal degeneration singularities in the affine Grassmannians of simple simply-laced algebraic groups are determined to be either Kleinian singularities of type A, or closures of minimal orbits in nilpotent cones. The singularities for non-simply-laced types are studied by intersection cohomology and equivariant Chow group methods.
\end{abstract}

\section{INTRODUCTION}

1.1. In the early 1980s H. Kraft and C. Procesi 9] classified the minimal degeneration singularities in the nilpotent cones of classical Lie algebras stratified by the adjoint orbits. We say that a pair of strata $\mathcal{O}, \mathcal{O}^{\prime}$ in a variety stratified by orbits of an algebraic group is a minimal degeneration if $\mathcal{O} \subset \overline{\mathcal{O}}^{\prime}$, and if $\mathcal{O} \subseteq \overline{\mathcal{O}}^{\prime \prime} \subseteq \overline{\mathcal{O}}^{\prime}$, then either $\mathcal{O}=\mathcal{O}^{\prime \prime}$ or $\mathcal{O}^{\prime \prime}=\mathcal{O}^{\prime}$. The normal singularities of this sort turn out to be smoothly equivalent to either Kleinian singularities of type A and D, or the closures of minimal orbits. A similar problem for some flag varieties was more recently investigated by M. Brion and P. Polo 4 .

1.2. In this paper we study the minimal degeneration singularities for the $G[[z]]$ orbits in the affine Grassmannians. More precisely, let $G$ be a simple finite dimensional algebraic group over an algebraically closed field $\mathbf{k}$ of characteristic 0 , and let $\mathcal{G}_{G}$ be the affine Grassmannian of $G$. Using a Levi subgroup technique we obtain the following.

Theorem A. If $G$ is of simply-laced type, then all singularities of the minimal degeneration singularities of $G[[z]]$-orbits in $\mathcal{G}_{G}$ are either Kleinian singularities of type $A$ or minimal singularities (closures of the minimal nilpotent orbits) of types corresponding to Dynkin subdiagrams of the Dynkin diagram of $G$.

1.3. For the non-simply-laced groups the situation is more complicated. In addition to Kleinian and minimal singularities, we obtain singularities which we call quasi-minimal. These singularities are studied by the methods of intersection cohomology [15, and equivariant multiplicities, 11, 3].

1.4. Our studies yield a completely new proof of the following result.

Corollary B. The smooth locus of the closure of a $G[[z]]$-orbit in $\mathcal{G}_{G}$ is precisely the $G[[z]]$-orbit itself.

This result is due to S. Evens and I. Mirković, it follows immediately from [5. Theorem 0.1(b)] which describes the characteristic cycle of the intersection cohomology sheaf on the closure of a $G[[z]]$-orbit. 
1.5. The paper is organized as follows. After introducing our notation and conventions in the Section 2 we prove a "Levi Lemma" dealing with the action of the loop group of a Levi subgroup of $G$ on $\mathcal{G}_{G}$ in the Section 3 .

In Section 4 we cite a crucial result of J. Stembrige 22] describing the minimal degeneration of coweights indexing the $G(O)$-orbits.

We then apply the Levi Lemma to describe some minimal degeneration singularities and prove Theorem A in the Section 5

Calculations of intersection cohomology dimensions in the Section [ give us some information on the rational smoothness of our singularities.

In Section 7 we study the singularities appearing in the rank 2 cases by methods of 11,3 .

Finally, we deduce Corollary B in the section 8

Acknowledgment. We are grateful to A. Braverman, M. Finkelberg, S. Kumar, I. Mirković, and J. Starr for very useful discussions. The research of A. M. and M. V. was supported by the NSF Postdoctoral Research Fellowships. The research of V. O. was partially supported by NSF grant DMS-0098830.

\section{Preliminaries}

\subsection{Notation.}

2.1.1. Let $G$ be a simple finite dimensional algebraic group defined over an algebraically closed field $\mathbf{k}$ of characteristic 0 .

Let us fix a Borel subgroup and a maximal torus $T \subset B \subset G$. We will introduce the following notation and conventions:

(2.1.1) $P \subset G$ is a paprabolic subgroup containing $B$.

(2.1.2) $L_{P}=P / N_{P}$ is the Levi quotient of $P$ by its nilpotent radical $N_{P}$.

(2.1.3) $M=\left[L_{P}, L_{P}\right]$ is the commutant of $L_{P} . M$ is a connected semisimple group. We will consider $M$ as a subgroup of $G$.

(2.1.4) $I$ is the set of vertices of the Dynkin diagram associated to $G$. The simple roots of the corresponding root system are denoted by $\alpha_{i}$ for $i \in I$, and the simple coroots by $\check{\alpha}_{i}$ for $i \in I$.

(2.1.5) $\mathrm{Q}_{G}$ is the coroot lattice of $G$ and $\mathrm{Q}_{\bar{G}}^{\geq 0}$ is the nonnegative cone in $\mathrm{Q}_{G}$.

(2.1.6) $\Lambda_{G}$ is the coweight lattice of $G$. The fundamental coweights are denoted by $\check{\omega}_{i}$ for $i \in I$. The partial order on the set of coweights: $\mu \geq \lambda$ if and only if $\mu-\lambda \in \mathrm{Q}_{\bar{G}}^{\geq 0}$.

2.1.2. From now on we will assume that the parabolic $P$ is associated to a connected Dynkin subdiagram of the Dynkin diagram of $G$. Then $M$ is a simple group of type described by this Dynkin subdiagram. We will denote the set of vertices of the subdiagram by $I_{M}$. We need some more notation:

(2.1.1) $\mathrm{Q}_{M} \subseteq Q_{G}$ is the coroot lattice of $M$, and $\Lambda_{M} \subseteq \Lambda_{G}$ is the coweight lattice of $M$.

(2.1.2) Let $\lambda, \mu \in \Lambda_{G}$. Then

$$
\lambda=\sum_{i \in I} \lambda_{i} \check{\omega}_{i} \quad \text { and } \quad \mu=\sum_{i \in I} \mu_{i} \check{\omega}_{i} .
$$


THE MINIMAL DEGENERATION SINGULARITIES IN THE AFFINE GRASSMANNIANS 3

We denote:

$$
\lambda_{M}=\sum_{i \in I_{M}} \lambda_{i} \check{\omega}_{i} \quad \text { and } \quad \mu=\sum_{i \in I_{M}} \mu_{i} \check{\omega}_{i}
$$

\subsection{Affine Grassmanians.}

2.2.1. Let $O=\mathbf{k}[[z]]$ be the ring of formal power series and $K=\mathbf{k}((z))$ be its field of fractions. The affine Grassmannian $\mathcal{G}_{G}$ is the ind-scheme whose k-points are given by $G(K) / G(O)$.

We will recall here some facts about affine Grassmannians mostly borrowed from [1, 2, 6, 12, 20, 17, where we refer the reader for many more details.

2.2.2. The coweight $\lambda \in \Lambda_{G}$ may be considered as an element of $\mathcal{G}_{G}$ via the identification $\Lambda_{G}=\operatorname{Hom}\left(\mathbf{k}^{*}, T\right)=T(K) / T(O)$. It is well known that the $G(O)$ orbits on $\mathcal{G}_{G}$ are indexed by dominant coweights. The notation:

$$
\mathcal{G}_{\lambda}=G(O) \cdot \lambda \text { for } \lambda \in \Lambda_{G}^{+} .
$$

It is well known that $\mathcal{G}_{\lambda} \subseteq \overline{\mathcal{G}}_{\mu}$ if and only if $\lambda \leq \mu$, and that $\operatorname{dim} \mathcal{G}_{\lambda}=\langle 2 \rho, \lambda\rangle$, where $2 \rho$ is the sum of positive roots.

2.2.3. Let us consider the group ind-scheme $G\left(\mathbf{k}\left[z^{-1}\right]\right)$, and let $L^{<0} G$ be the kernel of the map $G\left(\mathbf{k}\left[z^{-1}\right]\right) \rightarrow G$ defined by $z^{-1} \mapsto 0$. The following Lemma is well known.

Lemma. Consider $\lambda \in \Lambda_{G}^{+}$. The orbit $L^{<0} G \cdot \lambda$ is a transverse slice to $\mathcal{G}_{\lambda}$ at the point $\lambda$. In other words:

(i) $L^{<0} G \cdot \lambda$ is locally closed in $\mathcal{G}_{G}$.

(ii) The action map $G(O) \times\left(L^{<0} G \cdot \lambda\right) \rightarrow \mathcal{G}_{G}$ is an open embedding.

(iii) For any $\lambda \leq \mu \in \Lambda_{G}^{+}$

$$
\operatorname{dim}\left(L^{<0} G \cdot \lambda\right) \cap \overline{\mathcal{G}}_{\mu}=\operatorname{dim} \mathcal{G}_{\mu}-\operatorname{dim} \mathcal{G}_{\lambda} .
$$

Proof. (i) is clear. (ii) According to [20 Lemme 2.1] the multiplication morphism $G(O) \times L^{<0} G \rightarrow G(K)$ is an open embedding. Then the action morphism $G(O) \times$ $\left(L^{<0} G \cdot \lambda\right) \rightarrow \mathcal{G}_{G}$ is an open embedding and therefore is étale. This also proves (iii).

Lemma. Let $\lambda \leq \mu$ be two dominant coweights. The scheme $\left(L^{<0} G \cdot \lambda\right) \cap \overline{\mathcal{G}}_{\mu}$ is reduced, irreducible and normal.

Proof. Since $\left(L^{<0} G \cdot \lambda\right) \cap \overline{\mathcal{G}}_{\mu}$ is a transverse slice to $\mathcal{G}_{\lambda}$, and $\overline{\mathcal{G}}_{\mu}$ is a normal variety 10, 16, 7, 13, the variety $\left(L^{<0} G \cdot \lambda\right) \cap \overline{\mathcal{G}}_{\mu}$ is also normal and irreducible.

2.2.4. We will need one more lemma on the $\mathbf{k}^{*}$-action. The group $\mathbf{k}^{*}$ acts on $\mathcal{G}_{G}$ by "loop rotations"

$$
z \mapsto s z \quad \text { for } s \in \mathbf{k}^{*} .
$$

The following Lemma is lifted from [19].

Lemma. We have:

(i) $L^{<0} G \cdot \lambda \cap \overline{\mathcal{G}}_{\mu}$ is $\mathbf{k}^{*}$-invariant.

(ii) $L \in L^{<0} G \cdot \lambda$ if and only if $\lim _{s \rightarrow \infty} L=\lambda$.

\subsection{Kleinian and minimal singularities.}


2.3.1. We will think of a Kleinian singularity of type $A_{p}$ as an invariant theory quotient

$$
\mathbf{k}^{2} /(\mathbb{Z} /(p+1))
$$

of the affine space $\mathbf{k}^{2}$ by the cyclic group of order $p+1$, cf. [21, III.6.1].

2.3.2. Let $\mathfrak{g}=\operatorname{Lie} G$ be the Lie algebra of our group $G$. Let $\alpha_{\max }$ be the maximal root. It is well known that $\check{\alpha}_{\max }$ is the short dominant coroot. Let $v_{\max } \in \mathfrak{g}_{\max }$ be a highest weight vector. The conjugacy class $\mathcal{O}_{\min }=\operatorname{Ad} G \cdot v_{\max }$ is the minimal nilpotent orbit, and its closure

$$
\overline{\mathcal{O}}_{\min }(\mathfrak{g})=\mathcal{O}_{\min } \sqcup\{0\}
$$

is called the minimal singularity of type $\mathfrak{g}$. We will also index the minimal singularities by the Dynkin diagrams corresponding to $\mathfrak{g}$ and by small letters corresponding to capital letters indicating the type of $\mathfrak{g}$. For example the minimal singularity of $s l_{3}$ may be referred to as either $\overline{\mathcal{O}}_{\text {min }}\left(s l_{3}\right)$ or $\overline{\mathcal{O}}_{\text {min }}(\circ-\circ)$ or minimal singularity of type $a_{2}$. For many more details we refer the reader to [8, 9].

2.3.3. Minimal singularities and affine Grassmannians. Let $\lambda=0$ and $\check{\alpha}_{\max }$ be the short dominant coroot. We are grateful to I. Mirković for explaining to us the following

Lemma. There exists an isomorphism of algebraic varieties.

$$
\left(L^{<0} G \cdot 0\right) \cap \overline{\mathcal{G}}_{\check{\alpha}_{\max }} \simeq \overline{\mathcal{O}}_{\min }(\mathfrak{g}) .
$$

Proof. By the construction of [2] Page 182] the variety

$$
\overline{\mathcal{G}}_{\check{\alpha}_{\text {max }}} \simeq\{0\} \sqcup \mathcal{O}_{\text {min }} \sqcup P\left(\mathcal{O}_{\text {min }}\right)
$$

is the disjoint union of $3 G$-orbits, where $P\left(\mathcal{O}_{\text {min }}\right) \subset P(\mathfrak{g})$ the the projectivization of $\mathcal{O}_{\text {min }}$. Now the variety $\left(L^{<0} G \cdot 0\right) \cap \overline{\mathcal{G}}_{\check{\alpha}_{\max }}$ is $G$-invariant, and therefore a union of $G$-orbits. Since $P\left(\mathcal{O}_{\text {min }}\right)$ is $\mathbf{k}^{*}$-invariant, closed, and separated from 0 , by Lemma 2.2.4 we have

$$
\left(L^{<0} G \cdot 0\right) \cap \overline{\mathcal{G}}_{\check{\alpha}_{\max }} \simeq\{0\} \sqcup \mathcal{O}_{\min }=\overline{\mathcal{O}}_{\min } .
$$

\section{The Levi Lemma}

3.1. Let us consider two dominant coweights $\lambda \leq \mu \in \Lambda_{G}^{+}$such that their difference $\mu-\lambda \in \Lambda_{M}$ is in the coroot lattice of $M$ generated by $\check{\alpha}_{i}$ for $i \in I_{M}$ and therefore

$$
\mu-\lambda=\mu_{M}-\lambda_{M} \in \mathrm{Q}_{M},
$$

where $\lambda_{M}, \mu_{M}$ are defined in 2.1.2.1

3.1.1. Since we consider $M$ to be a subgroup of $G$, the group $M(K)$ acts on the affine Grassmannian $\mathcal{G}_{G}$.

Lemma. There exists a natural ind-scheme isomorphism

$$
M(K) \cdot \lambda \simeq \mathcal{G}_{M}
$$

given by the map $m \cdot \lambda \mapsto m \cdot \lambda_{M}$ for $m \in M(K)$. Moreover, this isomorphism restricts to the isomorphisms of varieties

$$
\mathcal{G}_{G} \supset\left(L^{<0} M \cdot \lambda\right) \cap \overline{M(O) \cdot \mu} \simeq\left(L^{<0} M \cdot \lambda_{M}\right) \cap \overline{M(O) \cdot \mu_{M}} \subset \mathcal{G}_{M} .
$$


THE MINIMAL DEGENERATION SINGULARITIES IN THE AFFINE GRASSMANNIANS 5

Proof. Indeed, $M(K) \cdot \lambda=M(K) / M(K)_{\lambda}$ where $M(K)_{\lambda}$ is the stabilizer of $\lambda$ in $M(K)$. Now

$$
\begin{aligned}
M(K)_{\lambda} & =M(K) \cap z^{\lambda} G(O) z^{-\lambda} \\
& =z^{\lambda}\left(z^{-\lambda} M(K) z^{\lambda} \cap G(O)\right) z^{-\lambda} \\
& =z^{\lambda}(M(K) \cap G(O)) z^{-\lambda} \\
& =z^{\lambda_{M}} M(O) z^{-\lambda_{M}} .
\end{aligned}
$$

3.1.2. We will need another

Lemma. Consider $\lambda \leq \mu \in \Lambda_{G}^{+}$with $\mu-\lambda \in \mathrm{Q}_{M}$. Then

$$
L^{<0} M \cdot \lambda \cap \overline{M(O) \cdot \mu}=L^{<0} G \cdot \lambda \cap \overline{G(O) \cdot \mu} \subset \mathcal{G}_{G} .
$$

Proof. Denote

$$
Y_{M}=L^{<0} M \cdot \lambda \cap \overline{M(O) \cdot \mu} \text { and } Y_{G}=L^{<0} G \cdot \lambda \cap \overline{G(O) \cdot \mu} .
$$

According to the lemma 2.2.4 both $Y_{M}$ and $Y_{G}$ are $\mathbf{k}^{*}$-invariant and therefore their closures $\bar{Y}_{M}$ and $\bar{Y}_{G}$ are also $\mathbf{k}^{*}$-invariant. Now due to the equation (3.1.0.1) and the Lemma 3.1.1 $\operatorname{dim} Y_{M}=\operatorname{dim} Y_{G}$. Thus $\bar{Y}_{M}=\bar{Y}_{G}$.

By construction, $Y_{M} \subseteq Y_{G}$. Now $\partial Y_{M}=\bar{Y}_{M}-Y_{M}$ is closed since $Y_{M}$ is locally closed.

Now, consider a point $p \in Y_{G}-Y_{M} \subset \partial Y_{M}$. According to the Lemma 2.2.4 $\mathbf{k}^{*}$ action retracts $p$ to $\lambda$ but this is impossible since $p$ is in a closed and $\mathbf{k}^{*}$-invariant set $\partial Y_{M}$ which does not contain $\lambda$. This contradiction shows that $Y_{G}-Y_{M} \cap \partial Y_{M}=\emptyset$.

Thus we have a bijective map of normal varieties $Y_{M} \rightarrow Y_{G}$ and thus an isomorphism.

Corollary. [Levi Lemma] There exists an isomorphism of algebraic varieties

$$
\mathcal{G}_{M} \supset L^{<0} M \cdot \lambda_{M} \cap \overline{M(O) \cdot \mu_{M}}=L^{<0} G \cdot \lambda \cap \overline{G(O) \cdot \mu} \subset \mathcal{G}_{G} .
$$

Proof. Follows from Lemma 3.1.1 and Lemma 3.1.2

\section{Minimal Degenerations of Coweights}

4.1. We will say that a pair $\lambda, \mu \in \Lambda_{G}^{+}$of dominant coweights is a minimal degeneration if

(i) $\lambda<\mu$,

(ii) if $\check{\nu} \in \Lambda_{G}^{+}$is such that $\lambda \leq \check{\nu} \leq \mu$ then either $\check{\nu}=\lambda$ or $\check{\nu}=\mu$.

The pair $\lambda, \mu$ which is a minimal degeneration will be denoted as $\mu \rightsquigarrow \lambda$.

4.2. All minimal degenerations $\mu \rightsquigarrow \lambda$ are classified by J. Stembridge. We will say that the support $\operatorname{supp}(\mu-\lambda)$ of $\mu-\lambda$ is the Dynkin subdiagram involving all simple coroots appearing in the decomposition of $\mu-\lambda$. It is obvious that for a minimal degeneration $\operatorname{supp}(\mu-\lambda)$ is connected. Here is the Stembridge's list, see [22, Theorem 2.8]:

Theorem. 22] The pair $\mu \rightsquigarrow \lambda$ is a minimal degeneration if and only if one of the following holds

(4.2.1) $\mu-\lambda$ is a simple coroot $\alpha_{i}, i \in I$. 
(4.2.2) $\mu-\lambda$ is the short dominant coroot of $\operatorname{supp}(\mu-\lambda)$ and $\lambda=0$ on $\operatorname{supp}(\mu-$ $\lambda)$.

(4.2.3) $\mu-\lambda$ is the short dominant coroot of $\operatorname{supp}(\mu-\lambda) ; \operatorname{supp}(\mu-\lambda)$ is of type $C_{n}$ and $\lambda$ on $\operatorname{supp}(\mu-\lambda)$ is

$$
0 \longleftarrow 0-0 \Longleftarrow 1
$$

(4.2.4) $\operatorname{supp}(\mu-\lambda)$ is of type $G_{2}$, and $\lambda=(2 \Longrightarrow 0), \mu=(1 \Longrightarrow 1)$ on $\operatorname{supp}(\mu-\lambda)$.

(4.2.5) $\operatorname{supp}(\mu-\lambda)$ is of type $G_{2}$, and $\lambda=(1 \Longrightarrow 0), \mu=(0 \Longrightarrow 1)$ on $\operatorname{supp}(\mu-\lambda)$.

\section{Minimal degenerations of $G(O)$-orbits and Levi subgroups}

\subsection{The $P G L_{2}$ case.}

5.1.1. In the $P G L_{2}$ case the pair $\lambda, \mu \in \Lambda_{G}^{+}$is a minimal degeneration if and only if $\mu=(p+2) \check{\omega}$ and $\lambda=p \check{\omega}$, where $\check{\omega}$ is the fundamental coweight and $p \geq 0$. In this case the Main Theorem of 18 implies the following

Lemma. Let $G=P G L_{2}, \lambda=p \check{\omega}$ and $\mu=(p+2) \check{\omega}$. Then the singularity of the Schubert variety $\overline{\mathcal{G}}_{\mu}$ along $\mathcal{G}_{\lambda}$ is a Kleinian singularity of type $A_{p+1}$. More precisely,

$$
\left(L^{<0} G \cdot \lambda\right) \cap \overline{\mathcal{G}}_{\mu} \simeq \mathbf{k}^{2} /(\mathbb{Z} /(p+2)) .
$$

Proof. It is shown in 18 that $\left(L^{<0} G \cdot \lambda\right) \cap \overline{\mathcal{G}}_{\mu}$ is isomorphic to a transverse slice to the subregular orbit in the nilpotent cone $\mathcal{N} \subset s l_{p+2}$. The statement follows by a celebrated result of Brieskorn and Slodowy [21]. For $p=0$ the lemma follows already from [14].

\subsection{The proof of Theorem A.}

Theorem. Let $\lambda, \mu \in \Lambda_{G}^{+}$be two dominant coweights, let $\lambda=\sum_{i \in I} \lambda_{i} \check{\omega}_{i}$, and let $\mu \rightsquigarrow \lambda$ be a minimal degeneration. Then

(5.2.1) If $\mu-\lambda=\check{\alpha}_{i}$ is a simple coroot for $i \in I$, then

$$
\left(L^{<0} G \cdot \lambda\right) \cap \overline{\mathcal{G}}_{\mu} \simeq \mathbf{k}^{2} /\left(\mathbb{Z} /\left(\lambda_{i}+2\right)\right)
$$

In other words, we have a Kleinian singularity of type $A_{\lambda_{i}+1}$.

(5.2.2) If $\mu-\lambda$ is the short dominant coroot of of the root system of type $\operatorname{supp}(\mu-\lambda)$ and $\lambda=0$ on $\operatorname{supp}(\mu-\lambda)$, then

$$
\left(L^{<0} G \cdot \lambda\right) \cap \overline{\mathcal{G}}_{\mu} \simeq \overline{\mathcal{O}}_{\min }(\operatorname{supp}(\mu-\lambda)),
$$

i.e., we have the minimal singularity of type $\operatorname{supp}(\mu-\lambda)$.

Proof. In the case (5.2.1) the Theorem follows from the Levi Lemma (Corollary 3.1.2) and Lemma 5.1.1 In the case (5.2.2) the Theorem follows from the Levi Lemma (Corollary 3.1.2) and Lemma 2.3.3. In the simply-laced case Theorem A follows. If $G$ is of type $A$ the theorem follows already from [18].

\section{INTERSECTION COHOMOLOGY CALCULATIONS}

\subsection{Notation.}


THE MINIMAL DEGENERATION SINGULARITIES IN THE AFFINE GRASSMANNIANS 7

6.1.1. Definition. We will say that a variety $X$ is rationally smooth at the point $x$ if the stalk of the intersection cohomology complex is 1-dimensional in degree $(-\operatorname{dim} X)$, i.e. $\mathbf{I H}_{x}(X)=\mathbf{k}[\operatorname{dim} X]$. We will say that $X$ is rationally smooth if it is rationally smooth at every point, i.e $\mathbf{I H}(X)$ is the constant sheaf shifted by $\operatorname{dim} X$.

6.1.2. By Lemma 2.2.3 if $\mu \rightsquigarrow \lambda$ is a minimal degeneration, the variety $\left(L^{<0} G\right.$. $\lambda) \cap \overline{\mathcal{G}}_{\mu}$ is smooth (and rationally smooth) at every point but $\lambda$. Let us denote:

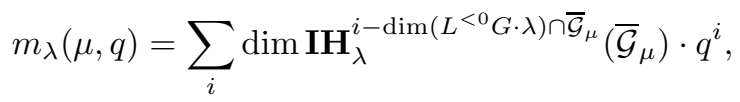

where $\mathbf{I H}^{i}\left(\overline{\mathcal{G}}_{\mu}\right)$ is the $i$-th cohomology sheaf of the intersection cohomology complex of $\mathcal{G}_{\mu}$ and $\mathbf{I H}_{\lambda}^{i}\left(\overline{\mathcal{G}}_{\mu}\right)$ is the stalk of $\mathbf{I} \mathbf{H}^{i}\left(\overline{\mathcal{G}}_{\mu}\right)$ at the point $\lambda \in \overline{\mathcal{G}}_{\mu}$. We will also consider the Euler characteristic

$$
\chi_{\lambda}(\mu)=m_{\lambda}(\mu, 1) .
$$

Clearly, $\left(L^{<0} G \cdot \lambda\right) \cap \overline{\mathcal{G}}_{\mu}$ is rationally smooth if and only if $m_{\lambda}(\mu)=\chi_{\lambda}(\mu)=1$.

6.1.3. Consider $\lambda$ and $\mu$ as the dominant weights for the Langlands dual group $G^{\vee}$ and let $m_{\lambda}(\mu)$ denote the multiplicity of the weight $\lambda$ in the simple $G^{\vee}$-module with the highest weight $\mu$. According to [15] one has

$$
\chi_{\lambda}(\mu)=m_{\lambda}(\mu) .
$$

\subsection{Rational smoothness of minimal degenerations.}

6.2.1. Let us study the rational smoothness of the variety $\left(L^{<0} G \cdot \lambda\right) \cap \overline{\mathcal{G}}_{\mu}$ in the four cases of the Theorem 4.2 using the formula (6.1.3.1).

Proposition. Let $\mu \rightsquigarrow \lambda$ be a minimal degeneration and

(6.2.1) let $\mu \rightsquigarrow \lambda$ be as in 4.2.1. Then

$$
m_{\lambda}(\mu, q)=1
$$

and the variety $\left(L^{<0} G \cdot \lambda\right) \cap \overline{\mathcal{G}}_{\mu}$ is rationally smooth.

(6.2.2) let $\mu \rightsquigarrow \lambda$ be as in 4.2.2 with $\lambda=0$. Then

$$
m_{\lambda}(\mu, q)= \begin{cases}\sum_{i=1}^{n} q^{e_{i}-1}, & \operatorname{supp}(\mu-\lambda) \text { of type } A D E, \\ \sum_{i=0}^{n-2} q^{2 i}, & \operatorname{supp}(\mu-\lambda) \text { of type } B_{n}, \\ 1, & \operatorname{supp}(\mu-\lambda) \text { of type } C_{n}, \\ 1+q^{4}, & \operatorname{supp}(\mu-\lambda) \text { of type } F_{4}, \\ 1, & \operatorname{supp}(\mu-\lambda) \text { of type } G_{2},\end{cases}
$$

where $e_{i} 1 \leq i \leq n$ are the exponents for types ADE. The Euler characteristic $\chi_{\lambda}(\mu)=n, n-1,1, n, 6,7,8,2,1$ for $\operatorname{supp}(\mu-\lambda)$ of type $A_{n}, B_{n}, C_{n}, D_{n}, E_{6}, E_{7}, E_{8}, F_{4}, G_{2}$ respectively. Thus the variety $\left(L^{<0} G \cdot \lambda\right) \cap \overline{\mathcal{G}}_{\mu}$ is rationally smooth in the cases $C_{n}$ and $G_{2}$.

(6.2.3) let $\mu \rightsquigarrow \lambda$ be as in 4.2.3. Then

$$
m_{\lambda}(\mu, q)=\sum_{i=0}^{n-1} q^{i}
$$

and $\chi_{\lambda}(\mu)=n$, and the variety $\left(L^{<0} G \cdot \lambda\right) \cap \overline{\mathcal{G}}_{\mu}$ is not rationally smooth. 
(6.2.4) let $G$ be of type $G_{2}$ and $\lambda=(2 \Longrightarrow 0)$, and $\mu=(1 \Longrightarrow 1)$. Then

$$
m_{\lambda}(\mu, q)=1+q
$$

and $\chi_{\lambda}(\mu)=2$ and the variety $\left(L^{<0} G \cdot \lambda\right) \cap \overline{\mathcal{G}}_{\mu}$ is not rationally smooth.

(6.2.5) let $G$ be of type $G_{2}$ and $\lambda=(1 \Longrightarrow 0), \mu=(0 \Longrightarrow 1)$. Then $m_{\lambda}(\mu, q)=\chi_{\lambda}(\mu)=1$ and the variety $\left(L^{<0} G \cdot \lambda\right) \cap \overline{\mathcal{G}}_{\mu}$ is rationally smooth.

Proof. First of all we apply the Levi Lemma (Corollary 3.1.2) to reduce the statements to the Levi subgroup associated to $\operatorname{supp}(\mu-\lambda)$. Then the formulas are obtained by the application of the results of [15] such as the formula [6.1.3.1), and the direct calculations.

\subsection{The quasi-minimal singularities.}

6.3.1. Type $a c_{n}$. Let us look again at the case 6.2.3 The singular variety $\left(L^{<0} G\right.$. $\lambda) \cap \overline{\mathcal{G}}_{\mu}$ has dimension $2 n$, the same as the minimal singularity of type $a_{n}$, and moreover, it follows from the Lemma that the polynomials $m_{\lambda}(\mu, q)$ coincide in our case and the case of the minimal singularity of type $a_{n}$ :

$$
m_{\lambda}(\mu, q)=m_{\lambda}^{a_{n}}(\mu, q)=\sum_{i=0}^{n-1} q^{i} .
$$

We will call this singularity arising from the affine Grassmannian of type $C_{n}$ the quasi-minimal singularity of type $a c_{n}$. Notice that according to Lemma 5.1.1 the singularity $a c_{1}$ is the Kleinian singularity of type $A_{2}$.

6.3.2. Type $a g_{2}$. Let us look again at the case 6.2.4 The singular variety $\left(L^{<0} G\right.$. $\lambda) \cap \overline{\mathcal{G}}_{\mu}$ is 4-dimensional as is the minimal singularity of type $a_{2}$ and moreover, it follows from the Lemma that the polynomials $m_{\lambda}(\mu, q)$ coincide in our case and the case of the minimal singularity of type $a_{2}$ :

$$
m_{\lambda}(\mu, q)=m_{\lambda}^{a_{2}}(\mu, q)=1+q .
$$

We will call the singularity arising from the affine Grassmannian the quasi-minimal singularity of type $a g_{2}$.

6.3.3. Type $c g_{2}$. Let us look again at the case 6.2.5 The singular variety $\left(L^{<0} G\right.$. $\lambda) \cap \overline{\mathcal{G}}_{\mu}$ is 4 -dimensional as is the minimal singularity of type $c_{2}$ and moreover it follows from the Lemma that both our variety and the minimal singularity of type $c_{2}$ are rationally smooth (but not smooth, see Section 17). We will call the singularity arising from the affine Grassmannian the quasi-minimal singularity of type $c g_{2}$.

\section{EQUiVARIANT MULTIPLICITIES IN RANK 2}

7.1. In this section we will study the rank 2 situation in more detail. Namely, for a minimal degeneration $\mu \rightsquigarrow \lambda$ the variety $\left(L^{<0} G \cdot \lambda\right) \cap \overline{\mathcal{G}}_{\mu}$ is invariant under the action of torus $T \times \mathbf{k}^{*}$ and the point $\lambda$ is fixed by this action. Here $T$ is the maximal torus of $G$ and $\mathbf{k}^{*}$ acts by loop rotations. Thus the equivariant multiplicity $e_{\lambda}(\mu)$ of the variety $\left(L^{<0} G \cdot \lambda\right) \cap \overline{\mathcal{G}}_{\mu}$ at the point $\lambda$ (i.e. the localization of the fundamental class in the $\left(T \times \mathbf{k}^{*}\right)$-equivariant Chow group at the only fixed point $\left.\lambda\right)$ is defined, see e.g. 3. Recall that $e_{\lambda}(\mu)$ is a rational function on the Lie algebra of the torus 
$T \times \mathbf{k}^{*}$. In this section we calculate $e_{\lambda}(\mu)$ in all rank 2 cases when the codimension of degeneration is $>2$.

Our calculation is performed as follows. Let $\mathfrak{F} l$ denote the affine flag variety of $G$ and let $\pi: \mathfrak{F} l \rightarrow \mathcal{G}$ be the canonical projection. The map $\pi$ is smooth with all of its fibers isomorphic to the finite dimensional flag variety $G / B$. Let $\mathcal{G}_{\lambda} \subset \overline{\mathcal{G}}_{\mu}$ be a minimal degeneration. Then the singularity $\pi^{-1}\left(\mathcal{G}_{\lambda}\right) \subset \pi^{-1}\left(\overline{\mathcal{G}}_{\mu}\right)$ is smoothly equivalent to the singularity $\mathcal{G}_{\lambda} \subset \overline{\mathcal{G}}_{\mu}$. Recall that the Schubert varieties in $\mathfrak{F} l$ are labeled by the elements of the (extended) affine Weyl group $W_{a f f}$. Let $y \in W_{a f f}$ (respectively $w \in W_{a}$ ) label the open Schubert variety $X_{y}$ in $\mathcal{G}_{\lambda}$ (respectively $\mathcal{G}_{\mu}$ ). A formula for calculation of the equivariant multiplicity $e_{y} X(w)$ is given in [3] and one deduces easily from this a formula for equivariant multiplicity $e_{\lambda}(\mu)$ of the transversal slice, see [3 page 27] We perform our calculations using this formula and a computer.

7.2. Kumar's Criterion. In 11] S. Kumar gave a general criterion for smoothness of Schubert varieties of a general Kac-Moody group in terms of equivariant multiplicities. In particular, Kumar's criterion implies that if the variety $\mathcal{G}_{\lambda} \subset \overline{\mathcal{G}}_{\mu}$ is smooth at $\lambda$ then

$$
e_{\lambda}(\mu)=\prod_{\alpha \in S} \alpha^{-1}
$$

where $S$ is a certain finite subset of the set of roots. We will see that Kumar's criterion and our calculations imply that in all cases considered in this section the varieties $\left(L^{<0} G \cdot \lambda\right) \cap \overline{\mathcal{G}}_{\mu}$ are not smooth.

7.3. Notation. In our calculations below we denote the simple roots by $\alpha_{i}, i=$ $0,1,2$; the simple reflection corresponding to $\alpha_{i}$ is denoted by $s_{i} ; s_{0}$ always denotes the affine simple reflection.

\subsection{Type $A_{2}$.}

7.4.1. The affine Weyl goup $W_{a f f}\left(A_{2}\right)$ of type $A_{2}$ is described as follows

$$
W_{a f f}\left(A_{2}\right)=\left\{s_{0}, s_{1}, s_{2} \mid\left(s_{1} s_{2}\right)^{3}=1,\left(s_{0} s_{1}\right)^{3}=1,\left(s_{0} s_{2}\right)^{3}=1\right\} .
$$

There is only one minimal degeneration:

7.4.2. Minimal singularity of type $a_{2}$. This is a singularity of codimension 4 . We have

$$
w=s_{1} s_{2} s_{1} s_{0} s_{1} s_{2} s_{1}, \quad y=s_{1} s_{2} s_{1} .
$$

The equivariant multiplicity:

$$
e_{\lambda}(\mu)=\frac{2\left(3 \alpha_{0}^{2}+6 \alpha_{0} \alpha_{1}+2 \alpha_{1}^{2}+6 \alpha_{0} \alpha_{2}+5 \alpha_{1} \alpha_{2}+2 \alpha_{2}^{2}\right)}{\alpha_{0}\left(\alpha_{0}+\alpha_{1}\right)\left(\alpha_{0}+\alpha_{2}\right)\left(\alpha_{0}+2 \alpha_{1}+\alpha_{2}\right)\left(\alpha_{0}+\alpha_{1}+2 \alpha_{2}\right)\left(\alpha_{0}+2 \alpha_{1}+2 \alpha_{2}\right)}
$$

\subsection{Type $C_{2}$.}

7.5.1. The affine Weyl group $W_{a f f}\left(C_{2}\right)$ of type $C_{2}$ is described as follows

$$
W_{a f f}\left(C_{2}\right)=\left\{s_{0}, s_{1}, s_{2} \mid\left(s_{1} s_{2}\right)^{4}=1,\left(s_{0} s_{2}\right)^{4}=1,\left(s_{0} s_{1}\right)^{2}=1\right\} .
$$

There are two minimal degenerations: 
7.5.2. Minimal singularity of type $c_{2} \cdot 0 \Longrightarrow 1 \rightsquigarrow 0 \Longrightarrow 0$ This is s singularity of codimension 4 . In this case

$$
w=s_{2} s_{1} s_{2} s_{1} s_{0} s_{2} s_{1} s_{2}, \quad y=s_{2} s_{1} s_{2} s_{1} .
$$

The equivariant multiplicity:

$$
e_{\lambda}(\mu)=\frac{8}{\alpha_{0}\left(\alpha_{0}+2 \alpha_{2}\right)\left(\alpha_{0}+2 \alpha_{1}+2 \alpha_{2}\right)\left(\alpha_{0}+2 \alpha_{1}+4 \alpha_{2}\right)}
$$

7.5.3. Quasi-minimal $a c_{2} .1 \Longrightarrow 1 \rightsquigarrow 1 \Longrightarrow 0$ This is s singularity of codimension 4 . In this case

$$
w=s_{2} s_{1} s_{2} s_{1} s_{0} s_{2} s_{0} s_{1} s_{2} s_{0} s_{2}, \quad y=s_{2} s_{1} s_{2} s_{1} s_{0} s_{2} s_{0} .
$$

The equivariant multiplicity:

$$
e_{\lambda}(\mu)=\frac{2\left(11 \alpha_{0}^{2}+21 \alpha_{0} \alpha_{1}+9 \alpha_{1}^{2}+43 \alpha_{0} \alpha_{2}+39 \alpha_{1} \alpha_{2}+36 \alpha_{2}^{2}\right)}{\alpha_{0}\left(\alpha_{0}+\alpha_{1}+\alpha_{2}\right)\left(\alpha_{0}+2 \alpha_{2}\right)\left(\alpha_{0}+\alpha_{1}+3 \alpha_{2}\right)\left(2 \alpha_{0}+3 \alpha_{1}+4 \alpha_{2}\right)\left(2 \alpha_{0}+3 \alpha_{1}+6 \alpha_{2}\right)}
$$

\subsection{Type $G_{2}$.}

7.6.1. The affine Weyl group $W_{a f f}\left(G_{2}\right)$ of type $G_{2}$ is described as follows

$$
W_{a f f}\left(G_{2}\right)=\left\{s_{0}, s_{1}, s_{2} \mid\left(s_{1} s_{2}\right)^{6}=1,\left(s_{0} s_{1}\right)^{3}=1,\left(s_{0} s_{2}\right)^{2}=1\right\} .
$$

There are three minimal degenerations:

7.6.2. Minimal singularity of type $g_{2} .1 \Longrightarrow 0 \rightsquigarrow 0 \Longrightarrow 0$. This is a singularity of codimension 6 . We have

$$
w=s_{2} s_{1} s_{2} s_{1} s_{2} s_{1} s_{0} s_{1} s_{2} s_{1} s_{2} s_{1}, \quad y=s_{2} s_{1} s_{2} s_{1} s_{2} s_{1} .
$$

The equivariant multiplicity:

$$
e_{\lambda}(\mu)=\frac{18}{\alpha_{0}\left(\alpha_{0}+\alpha_{1}\right)\left(\alpha_{0}+\alpha_{1}+3 \alpha_{2}\right)\left(\alpha_{0}+3 \alpha_{1}+3 \alpha_{2}\right)\left(\alpha_{0}+3 \alpha_{1}+6 \alpha_{2}\right)\left(\alpha_{0}+4 \alpha_{1}+6 \alpha_{2}\right)}
$$

7.6.3. Quasi-minimal of type $a g_{2} .1 \Longrightarrow 1 \rightsquigarrow 2 \Longrightarrow 0$. This is a singularity of codimension 4 . We have

$$
w=s_{2} s_{1} s_{2} s_{1} s_{2} s_{1} s_{0} s_{1} s_{2} s_{1} s_{2} s_{0} s_{1} s_{2} s_{1} s_{0} s_{2} s_{1} s_{2} s_{1} s_{2} s_{1},
$$

and

$$
y=s_{2} s_{1} s_{2} s_{1} s_{2} s_{1} s_{0} s_{1} s_{2} s_{1} s_{2} s_{1} s_{0} s_{1} s_{2} s_{1} s_{2} s_{1} .
$$

The equivariant multiplicity:

$$
e_{\lambda}(\mu)=\frac{2\left(27 \alpha_{0}^{2}+106 \alpha_{0} \alpha_{1}+103 \alpha_{1}^{2}+159 \alpha_{0} \alpha_{2}+309 \alpha_{1} \alpha_{2}+216 \alpha_{2}^{2}\right)}{\left(\alpha_{0}+\alpha_{1}\right)\left(\alpha_{0}+2 \alpha_{1}+2 \alpha_{2}\right)\left(\alpha_{0}+\alpha_{1}+3 \alpha_{2}\right)\left(\alpha_{0}+2 \alpha_{1}+4 \alpha_{2}\right)\left(3 \alpha_{0}+7 \alpha_{1}+9 \alpha_{2}\right)\left(3 \alpha_{0}+7 \alpha_{1}+12 \alpha_{2}\right)}
$$

7.6.4. Quasi-minimal singularity of type $c g_{2} \cdot 0 \Longrightarrow 1 \rightsquigarrow 1 \Longrightarrow 0$. This is a singularity of codimension 4 . We have

$$
w=s_{2} s_{1} s_{2} s_{1} s_{2} s_{1} s_{0} s_{1} s_{2} s_{1} s_{2} s_{0} s_{1} s_{2} s_{1} s_{2}, \quad y=s_{2} s_{1} s_{2} s_{1} s_{2} s_{1} s_{0} s_{1} s_{2} s_{1} s_{2} s_{1} .
$$

The equivariant multiplicity:

$$
e_{\lambda}(\mu)=\frac{27}{\left(\alpha_{0}+\alpha_{1}\right)\left(\alpha_{0}+\alpha_{1}+3 \alpha_{2}\right)\left(2 \alpha_{0}+5 \alpha_{1}+6 \alpha_{2}\right)\left(2 \alpha_{0}+5 \alpha_{1}+9 \alpha_{2}\right)}
$$


THE MINIMAL DEGENERATION SINGULARITIES IN THE AFFINE GRASSMANNIANS 11

7.7. Quasi-minimal singularities revisited. Recall that the singularities $a_{2}$, $a c_{2}, a g_{2}, c_{2}, c g_{2}$ have codimension 4 . Moreover one observes that the intersection cohomology of singularities of type $a_{2}, a c_{2}, a g_{2}$ (and, similarly, of $c_{2}$ and $c g_{2}$ ) are the same. We conjecture that all these singularities are pairwise smoothly non-equivalent. One verifies that the equivariant multiplicities $e_{\lambda}(\mu)$ are pairwise distinct (up to linear changes of coordinates with rational coefficients). This implies that at least the singularities above are different as singularities with torus action.

Similarly, the singularities of types $a_{n}$ and $a c_{n}$ have the same codimensions and intersection cohomology but we conjecture that these singularities are not smoothly equivalent.

\section{Smooth Locus}

8.1. Finally we use our results to prove the Evens-Mirković Theorem (Corollary B). Let us denote by $\overline{\mathcal{G}}_{\mu}^{\text {smooth }}$ the smooth locus of the Schubert variety $\overline{\mathcal{G}}_{\mu}$.

Corollary. For any dominant coweight $\lambda \in \Lambda_{G}^{+}$we have

$$
\overline{\mathcal{G}}_{\mu}^{\text {smooth }}=\mathcal{G}_{\mu} \text {. }
$$

Proof. It is enough to check that $\overline{\mathcal{G}}_{\mu}$ is singular along every irreducible component of the boundary $\overline{\mathcal{G}}_{\mu}-\mathcal{G}_{\mu}$. These irreducible components are precisely Schubert varieties $\overline{\mathcal{G}}_{\lambda}$ for all minimal degenerations $\mu \rightsquigarrow \lambda$.

Thus we have to check that the minimal degenerations of $G(O)$-orbits are singular for all cases in the Stembridge's list, see Theorem 4.2

In the cases 4.2 .1 and 4.2 .2 it follows from the Theorem 5.2

In the cases 4.2 .3 and 4.2 .4 the variety $\left(L^{<0} G \cdot \lambda\right) \cap \overline{\mathcal{G}}_{\mu}$ is not rationally smooth by Proposition 6.2.1 and therefore not smooth.

In the case 4.2 .5 the variety $\left(L^{<0} G \cdot \lambda\right) \cap \overline{\mathcal{G}}_{\mu}$ is singular by the Kumar's criterion, see formula (7.6.4.1).

\section{REFERENCES}

[1] A. Beauville and Y. Laszlo, Conformal blocks and generalized theta functions, Comm. Math. Phys. 164 (1994), no. 2, 385-419.

[2] A. Beilinson and V. Drinfeld, Quantization of Hitchin's integrable system and Hecke eigensheaves, preprint.

[3] M. Brion, Equivariant cohomology and equivariant intersection theory, Notes by Alvaro Rittatore. NATO Adv. Sci. Inst. Ser. C Math. Phys. Sci., 514, Representation theories and algebraic geometry (Montreal, PQ, 1997), 1-37.

[4] M. Brion and P. Polo, Generic singularities of certain Schubert varieties, Math. Z. 231 (1999), no. $2,301-324$.

[5] S. Evens and I. Mirković, Characteristic cycles for the loop Grassmannian and nilpotent orbits, Duke Math. J. 97 (1999), no. 1, 109-126.

[6] G. Faltings, A proof for the Verlinde formula, J. Algebraic Geometry 3 (1994), 347-347.

[7] G. Faltings, Algebraic loop groups and moduli spaces of bundles, J. Eur. Math. Soc. 5 (2003) 1, 41-68.

[8] H. Kraft and C. Procesi, Minimal singularities in $\mathrm{GL}_{n}$, Invent. Math. 62 (1981), no. 3, $503-515$.

[9] H. Kraft and C. Procesi, On the geometry of conjugacy classes in classical groups, Comment. Math. Helv. 57 (1982), no. 4, 539-602.

[10] S. Kumar, Demazure character formula in arbitrary Kac-Moody setting, Invent. Math. 89 (1987), no. 2, 395-423.

[11] S. Kumar, The nil Hecke ring and singularity of Schubert varieties, Invent. Math. 123 (1996), no. 3, 471-506. 
[12] Y. Laszlo and C. Sorger, The line bundles on the moduli of parabolic G-bundles over curves and their sections, Ann. Sci. École Norm. Sup. (4) 30 (1997), no. 4, 499-525.

[13] G. Littelmann, Conracting modules and standard monomial theory for symmetrizable KacMoody algebras, J. Amer. Math. Soc. 11 (1998), no. 3, 551-567.

[14] G. Lusztig, Green polynomials and singularities of unipotent classes, Adv. in Math. 42 (1981), no. $2,169-178$.

[15] G. Lusztig, Singularities, character formulas, and a q-analog of weight multiplicities, Analysis and topology on singular spaces, II, III (Luminy, 1981), 208-229, Astérisque, 101-102, Soc. Math. France, Paris, 1983.

[16] O. Mathieu, Formules de caractères pour les algèbres de Kac-Moody générales, Astèrisque No. 159-160 (1988), 267 pp.

[17] I. Mirković and K. Vilonen, Perverse sheaves on affine Grassmannians and Langlands duality, Math. Res. Lett. 7 (2000), no. 1, 13-24.

[18] I. Mirković and M. Vybornov, On quiver varieties and affine Grassmannians of type A, C. R. Acad. Sci. Paris, Ser. I (2003) 336 (3) 207-212.

[19] I. Mirković and M. Vybornov, Quiver varieties and Beilinson-Drinfeld Grassmannians of type A, preprint, 2002.

[20] B. C. Ngô and P. Polo, Résolutions de Demazure affines et formule de Casselman-Shalika géométrique, J. Algebraic Geom. 10 (2001), no. 3, 515-547.

[21] P. Slodowy, Simple singularities and simple algebraic groups, Lecture Notes in Mathematics, 815. Springer, Berlin, 1980.

[22] J. Stembridge, The partial order of dominant weights, Adv. Math. 136 (1998), no. 2, 340-364.

Department of Mathematics, Mit, 77 Massachusetts Avenue, Cambridge, MA 02139

E-mail address: malkin@math.mit.edu

E-mail address: ostrik@math.mit.edu

E-mail address: vybornov@math.mit.edu 